\title{
CORRIGENDUM
}

\section{Selecting native perennial plants for ecological intensification in Mediterranean greenhouse horticulture - CORRIGENDUM}

\author{
E. Rodríguez, M. González, D. Paredes, M. Campos and \\ E. Benítez
}

doi:10.1017/S0007485317001237 Published by Cambridge University Press, 4 December 2017.

The authors wish to apologise for errors within this article. The following is a list of the errors and corrections required.

\section{Affiliation}

${ }^{2}$ Cajamar Experimental Station

Introduction

Page 2, text line 47: 'direst' should be replaced by 'direct'

Material and methods

Site description

Page 2, text line 2: "Experimental Station 'Las Palmerillas'" should be replaced by "Cajamar Experimental Station"

Results

Identifying perennial plants harbouring pests

Page 4, text line 3: Dodonaea replaced by Dittrichia

Page 4, text line 19: Grimpoteuthis replaced by Genista

Page 4, text line 27: Rubus occidentalis replaced by Romero officinalis

Page 4, text line 32:Phanera replaced by Phlomis

Page 5, text line 1: Prunus replaced by Phillyrea

Page 5, text line 2: Marsdenia replaced by Macrochloa

Page 5, text line 3: Macrozamia replaced by Mirtus

Table 2:

The following row should have contained some data in bold, as follows:

Flowering $\times$ species

Sampling date

11761.48

3685.38

Table 3:

The following two rows should have contained some data in bold, as follows:

\begin{tabular}{|c|c|c|c|c|c|}
\hline Flowering $\times$ species & Sampling date & 786.25 & 449.67 & 1163.44 & 502.89 \\
\hline Prey $\times$ species & Sampling date & 608.42 & 427.69 & 1103.86 & 520.66 \\
\hline
\end{tabular}

\section{Reference}

Rodríguez, E., González, M., Paredes, D., Campos, M. and Benítez, E. Selecting native perennial plants for ecological intensification in Mediterranean greenhouse horticulture. Bulletin Ent Res. Published by Cambridge University Press, 4 December 2017. doi:10.1017/ S0007485317001237. 